\title{
True Chemical Composition Profiles at Polymer Interphases: Recovery from Measurements Distorted by Instrumental Broadening
}

\author{
PABLO TOMBA and GUILLERMO ELIÇABE* \\ Instituto de Investigaciones en Ciencia y Tecnología de Materiales (INTEMA) (CONICET-UNMdP), Facultad de Ingeniería, \\ Universidad Nacional de Mar del Plata, Juan B. Justo 4302, (7600) Mar del Plata. República Argentina (P.T., G.E.); and \\ Departamento de Ingeniería Química, Facultad de Ingeniería, Universidad Nacional de Mar del Plata, Juan B. Justo 4302, (7600) \\ Mar del Plata. República Argentina (P.T., G.E.)
}

\begin{abstract}
The determination of chemical composition profiles at polymer interphases is an important issue at the moment of elucidating the physical mechanisms that operate in polymer diffusion processes and for calculating diffusion parameters. Several techniques are available to measure these profiles, the most common being forward recoil spectroscopy, Rutherford backscattering spectrometry, nuclear reaction analysis, confocal Raman microspectroscopy (CRM), and scanning infrared microscopy. However, all these techniques are affected by the limited resolution of the experimental setup, which in practice produces a rounding effect on the sharp corners of the composition profile; this may lead to incorrect conclusions regarding the measurements. In this work an inverse technique is proposed to correct this undesirable effect in the profiles. The inversion is performed on a model of the measuring process, which includes the instrumental broadening function, a quantitative representation of the limited resolution. The proposed methodology was tested using numerically generated experiments and genuine experimental runs obtained from CRM measurements at interphases of polymer bilayers. In all cases, the recovered profiles were close to the expected ones. In the truly experimental results diffusion tails are observed behind and ahead of the diffusion front before the numerical treatment of the data. These tails may be caused by a genuine mass diffusion or by an artifact. After the numerical treatment the tails disappear and a sharp interphase is recovered, a result one expects for the polymer pairs under study.
\end{abstract}

Index Headings: Diffusion; Interphases; Computer modeling; Inverse problem; Raman spectroscopy.

\section{INTRODUCTION}

The experimental study of chemical composition profiles at interphases generated by polymer interdiffusion as well as by diffusion of small molecules in polymer matrices has received considerable attention in recent years. ${ }^{1-21}$ These studies have made possible the benchtesting of diffusion models and have contributed to the elucidation of the physical mechanisms that operate in polymer diffusion processes. ${ }^{2-11,13-16}$ Details of the measured composition profiles can be used to test physical models and to calculate diffusion parameters such as front advancing rates, profile slopes, and corner shapes. These parameters play a main role in fitting experimental data to models, and therefore, instrumental resolution and accuracy are crucial.

The chemical composition profile along the interphase diffusion path is the variable most often used to quantify results from diffusion studies because it can be experi-

Received 30 November 2002; accepted 31 March 2003.

* Author to whom correspondence should be sent. mentally measured for many systems. Much effort has been devoted to the experimental measurement of composition profiles at polymer interphases and many techniques have been developed for this purpose, but only a few are able to perform direct measurements. In the level of high spatial resolution (typically on the order of nanometers) forward recoil spectroscopy (FRES) and Rutherford backscattering spectrometry (RBS) have been by far the most widely employed to study composition profiles at polymer interphases in a direct form. ${ }^{1-11}$ Nuclear reaction analysis (NRA) has the same high intrinsic resolution, but only recently has it been applied to polymer studies. ${ }^{12-14}$ This group of techniques is based on hitting the sample surface with charged $\mathrm{He}$ nuclei $\left(\mathrm{He}^{2+}\right.$ for FRES or RBS, $\mathrm{He}^{3+}$ for NRA), and they require a heavier tracer element to be linked to the diffusing species. Collisions between $\mathrm{He}$ nuclei and the heavier element can produce a recoiling of the heavier element (in the case of FRES), backscattering of $\mathrm{He}^{2+}$ nuclei (in the case of RBS), or a nuclear reaction that produces $\mathrm{He}^{4+}$ ions (in the case of NRA). The energy of these particles depends on the depths from which they are produced, and the energy is recorded by an energy detector. The output of the energy detectors is fed into a multi-channel analyzer, which displays the number of particles versus energy. The RBS, FRES, or NRA energy spectra can be analyzed in a straightforward manner to yield the composition profile (chemical composition as a function of the depth) of the marked polymer in the sample.

Another group of techniques widely employed in the direct measurements of composition profiles at polymer interphases, but with lower spatial resolutions (on the order of micrometers), are vibrational spectroscopies. ${ }^{15-24}$ The confocal Raman microspectroscopy (CRM) technique has been shown to be a useful tool in the study of chemical compositions of very small volumes in polymeric samples. ${ }^{20,21}$ The confocal arrangement is based on a pinhole that, once a sample volume is irradiated with a laser beam, blocks the signal (Raman scattering) coming from out-of-focus planes, separating the signal from small sample elements (in-focus planes) from the signals produced by the surroundings. ${ }^{21}$ In this way, a profile of local chemical composition versus coordinate can easily be obtained. Based on a similar principle, scanning infrared microscopy (SIRM) has also been employed to study polymer interphases in direct form. This technique is penalized by a poor spatial resolution (hundreds of 
micrometers), limited by the slit opening and the optical arrangement used. ${ }^{23,24}$

Many sources may contribute to the broadening of the experimentally measured composition profiles; one of the most important of these sources are those attributed to instrumental factors. In the case of FRES, RBS, and NRA, the broadening in the energy values recorded by the detector has been reported as the most important. ${ }^{1-14}$ As the energy of the detected nuclei is a function of the depth into the polymer sample, it leads to a broadening in the diffusion coordinate scale. Many authors have used these techniques to study diffusion processes such as transport of small molecules into glassy polymer matrices and interpenetration of polymers with different physical properties..$^{1-11}$ They systematically report an artificial rounding of regions in the profiles where sharp corners are expected and attribute the effect to the instrumental broadening of the experimental technique.

In the case of CRM, many instrumental factors may contribute to the broadening of the composition profile. The performance of the confocal device has been reported as one of the most important. ${ }^{21}$ As the pinhole cannot completely block Raman scattering from out-of-focus planes, significant Raman scattering rising from the surroundings can also contribute to the local signal. Instrumental effects caused by refraction effects at the sample/ air interface are another very important factor, especially when depth-profiling studies are performed. The influence of the optical properties of the sample on the depth resolution has been mentioned in Refs. 17, 18, and 21, even though a more extensive analysis of this phenomenon can be found in Ref. 25. When the laser beam of the CRM is refracted at the air/sample interface, two separate instrumental effects need to be considered. In the first place, refraction increases the range of depths where Raman scattering is collected as one focuses deeper into the sample, and the instrumental resolution becomes much poorer (the deeper the poorer). Secondly, refraction at the sample/air interface causes the real focus (i.e., the region where local chemical composition is measured) to be placed deeper than the micrometric positioning screw indication; this geometric effect increases linearly with depth. ${ }^{25,26}$ However, refraction effects at the sample/air interface are not important when CRM is used to perform surface profiling studies. All of these effects lead to the rounding of the experimentally measured composition profile in regions where the composition profile is expected to present sharp corners. ${ }^{15-19}$

The same artifact has been reported when the interdiffusion between polymers with different molecular weights was studied using SIRM. ${ }^{23,24}$ The authors have attributed the artificial rounding of the profiles in the zones of steep edges to the finite width of the slit used in the optical device.

The broadening caused by instrumental factors over the measured composition profile is undesirable and almost impossible to eliminate experimentally. This broadening can be quantified by means of the instrumental broadening function (IBF). The artificial rounding of the composition profiles observed in the regions where sharp corners are expected is due to the shape of the IBF, typically a bell-shaped curve. Corrections of instrumental effects based on numerical techniques (convolution) have been proposed but are useful only when predictions from diffusion models are compared with experimental data. ${ }^{1,4-11}$

In this work, we propose a numerical strategy to recover the true composition profile at interphases, i.e., the corrected composition profile, free of the distortion caused by instrumental effects, once the IBF is known. The proposed solution is based on deconvoluting the experimentally measured composition profile with the previously known (or estimated) IBF. The problem posed falls in the category of inverse problems. In order to circumvent the ill-conditioned characteristic of this type of problem, regularization techniques are applied to guarantee the recovery of an accurate solution. In the next section, the explanation of the fundamentals of the technique is given. In the Results and Discussion section, some simulations are shown to illustrate the application of the technique, its limitations, and the criterion used to select the regularization parameter. Finally, we apply the technique to genuine experimental data distorted by instrumental broadening. They are obtained from CRM measurements at interphases of polymer bilayers where abrupt changes of chemical composition are known to exist.

\section{PROPOSED METHODOLOGY}

Inverse Problem Formulation. When two homogeneous layers that differ in their chemical composition are put in contact through a planar interface, the resulting composite material will have a chemical composition gradient in only one direction $(x)$, and a constant composition in the other directions $(y, z)$. In the case that interdiffusion between the layers is promoted (by elevating the temperature for a specified period of time), the original planar interface will be broadened. The true chemical composition profile present in the composite material along the $x$-axis direction is named $\varphi_{\mathrm{r}}(x)$.

We must take into account that the experimental techniques mentioned in the Introduction section commonly measure the experimental composition profiles in two modes. In the $A$ mode (SIRM and CRM) the chemical composition profile is determined by scanning the surface of the composite material along the $x$-axis, with the beam (laser or infrared) aligned in a direction normal to the $x$ axis. To perform the scanning of the surface, a crosssection of the composite material is needed, which sometimes requires sample microtoming. ${ }^{22-24}$ In the $B$ mode (FRES, RBS, NRA, or CRM in the depth profiling mode), the profile is measured by sampling the system through one of the layers of the composite material with the beam aligned in a direction parallel to the $x$-axis, which requires measurements below the sample surface.

In any of these cases, the experimental technique will measure a composition profile different from the true composition profile $\varphi_{\mathrm{r}}(x)$ due to the distortion produced by the instrument. We use the symbol $\varphi_{\mathrm{m}}(x)$ to designate the experimentally measured composition profile. The measured quantity $\varphi_{\mathrm{m}}(x)$ can be related to the true composition profile $\varphi_{\mathrm{r}}(x)$ through the following first-kind Fredholm integral equation: 


$$
\varphi_{\mathrm{m}}(x)=\int_{0}^{\infty} B(\mu, x) \varphi_{\mathrm{r}}(\mu) \mathrm{d} \mu
$$

The function $B(\mu, x)$ represents the IBF at a given point $x$. This IBF is normally a bell-shaped function that takes into account the contributions to the measured signal of the concentrations of the surroundings of the point with coordinate $x$.

To find $\varphi_{\mathrm{r}}(x)$ from Eq. 1, given $B(\mu, x)$ and the measured concentration profile $\varphi_{\mathrm{m}}(x)$, is not an easy task. Equation 1 is, to some extent that depends on the nature of the problem, ill conditioned. If no special precautions are taken, small errors in $\varphi_{\mathrm{m}}(x)$ produce greatly distorted solutions of $\varphi_{\mathrm{r}}(x)$. The so-called Philips-Thikonov technique $^{27}$ has proven to be a useful tool to solve Eq. 1 . With this technique, the solution is regularized so that the distortion introduced by the ill conditioning is minimized.

The problem can be practically solved if Eq. 1 is discretized in both variables, $x$ and $\mu$. Two vectors are defined:

$$
\begin{aligned}
\boldsymbol{\varphi}_{\mathrm{m}} & =\left[\boldsymbol{\varphi}_{\mathrm{m}}\left(x_{1}\right) \varphi_{\mathrm{m}}\left(x_{2}\right) \ldots \varphi_{\mathrm{m}}\left(x_{n_{1}}\right)\right]^{\mathrm{T}} \\
\boldsymbol{\varphi}_{\mathrm{r}} & =\left[\varphi_{\mathrm{r}}\left(x_{1}\right) \varphi_{\mathrm{r}}\left(x_{2}\right) \ldots \varphi_{\mathrm{r}}\left(x_{n_{2}}\right)\right]^{\mathrm{T}}
\end{aligned}
$$

Thus Eq. 1 may be computed approximately in discrete form as:

$$
\varphi_{\mathrm{m}} \approx \mathrm{B} \varphi_{\mathrm{r}}
$$

where $\mathbf{B}$ is a $\left(n_{1} \times n_{2}\right)$ matrix, whose values depend on the quadrature formula used to discretize Eq. 1. In Eq. 4 the equal sign may replace the approximate sign if a vector of measurement and discretization error $\mathbf{e}$ is included:

$$
\boldsymbol{\varphi}_{\mathrm{m}}=\mathbf{B} \boldsymbol{\varphi}_{\mathrm{r}}+\mathbf{e}
$$

Normally, discretization error may be reduced by proper selection of quadrature formula and discretization steps. Therefore, measurement error may only be considered important in Eq. 5. The solution of Eq. 5 using the Philips-Thikonov technique is given by:

$$
\boldsymbol{\varphi}_{\mathrm{r}}=\left(\mathbf{B}^{\mathrm{T}} \mathbf{B}+\gamma \mathbf{H}\right)^{-1} \mathbf{B}^{\mathrm{T}} \boldsymbol{\varphi}_{\mathrm{m}}
$$

where $\mathbf{H}=\mathbf{K}^{\mathrm{T}} \boldsymbol{\Gamma} \mathbf{K}$ is the regularization matrix. $\mathbf{K}$ is selected in most cases, as in Ref. 28. $\boldsymbol{\Gamma}$ is a weighting matrix that ponders the relative amount of regularization along the $x$ coordinate, and $\gamma$ is a parameter that ponders the amount of regularization on the sought solution, $\hat{\varphi}_{\mathrm{r}}$, compared to the amount of fitting of $\mathbf{B} \hat{\boldsymbol{\varphi}}_{\mathrm{r}} \equiv \hat{\boldsymbol{\varphi}}_{\mathrm{m}}$ to $\boldsymbol{\varphi}_{\mathrm{m}}$. For instance, if $\gamma=0$, no regularization is applied and then Eq. 6 coincides with the well-known least squares solution, $\hat{\boldsymbol{\varphi}}_{\mathrm{r}}=\left(\mathbf{B}^{\mathrm{T}} \mathbf{B}\right)^{-1} \mathbf{B}^{\mathrm{T}} \boldsymbol{\varphi}_{\mathrm{m}}$. However, because of the ill-conditioned nature of the problem, the least squares solution is unacceptable and a regularization parameter $\gamma$ different from zero must be selected. In what follows, it will be assumed that $\boldsymbol{\Gamma}$ is selected arbitrarily according to the concentration profile that is being processed, and $\gamma$ must be determined automatically from the measurements.

To compute $\gamma$ several methods have been proposed. In this work, the generalized cross validation (GCV) technique will be used. GCV is explained in detail elsewhere. ${ }^{29}$ The value of $\gamma$ computed by means of GCV is the one that minimizes the following function:

$$
V(\gamma)=\frac{\left|(\mathbf{I}-\mathbf{Z}) \boldsymbol{\varphi}_{\mathrm{m}}\right|^{2}}{|\operatorname{Trace}(\mathbf{I}-\mathbf{Z})|^{2}}
$$

where $\mathbf{Z}=\mathbf{B}\left(\mathbf{B}^{\mathrm{T}} \mathbf{B}+\gamma \mathbf{H}\right) \mathbf{B}^{\mathrm{T}}$ and $\mathbf{I}$ is the identity matrix.

Instrumental Broadening Function. In order to measure the chemical composition profile at the polymer interphase, the $x$-axis direction is generally scanned in one of the modes described above. The measured quantity at each point is not exactly the chemical composition at the point, but instead, due to the nature of the experimental technique, is an average of the composition of the surroundings, weighted in an inverse manner with the distance to the considered point. The exact manner in which the average is weighted determines the IBF that depends on the nature of the experimental technique; however, the following characteristics are commonly observed (with few exceptions) for the IBF: (1) it is a smooth and symmetrical bell-shaped type curve; and (2) it presents an almost invariant shape and spread with respect to the point where the chemical composition is measured. Under these assumptions, if the composition profile is measured well inside the sample, the IBF is such that the average is taken with the same weight on both sides of the focused point. In this case the area under the curve plotted by the broadening function must be equal to one to ensure a proper average. On the outer surface of the thin layer (where the first measurement is taken) and close to it, this condition must be modified because the IBF extends to regions where there is no material. In this case the IBF must be taken so that its area under the region where there is material is still one. After the previous considerations the broadening function can be expressed as:

$$
B(\mu, x)=\frac{b(\mu-x)}{\int_{0}^{\infty} b(\mu-x) \mathrm{d} \mu}=\phi(x) b(\mu-x)
$$

where $b(\mu-x)$ is a distribution-type symmetric function with area one. The dependence on the difference of the two arguments reflects the invariance of the shape of $b(\cdot)$ along the different positions on the measurement axis. This function, $b(\cdot)$, is weighted with $\phi(x)$, which is equal to two at $x=0$, i.e., on the surface of the thin layer, and tends to one for $x$ larger than the width of the distribution. In this form, the area under the curve plotted by $B(\mu, x)$ on the positive $\mu$ axis for $x \geq 0$, is always equal to one. Thus, Eq. 1 can be written as:

$$
\varphi_{\mathrm{m}}(x)=\phi(x) \int_{-\infty}^{x} b(\mu) \varphi_{\mathrm{r}}(x-\mu) \mathrm{d} \mu
$$

In order to characterize the IBF, Eq. 9 can be used to estimate $b(\mu)$ in the same manner than Eq. 1 was suggested to be used to estimate $\varphi_{\mathrm{r}}(x)$. Because the roles of $b(\mu)$ and $\varphi_{\mathrm{r}}(x)$ in Eqs. 1 and 9 are interchanged, the experimental determination must be performed on a sample in which the concentration profile is known. Another issue to consider is that $\phi(x)$ must be known; however, it depends on the unknown $b(\mu)$. This difficulty may be solved by using measurements far from the surface of the thin layer. In this case, i.e., for large $x, \phi(x) \rightarrow 1$ and Eq. 9 becomes: 


$$
\varphi_{\mathrm{m}}(x)=\int_{-\infty}^{x} b(\mu) \varphi_{\mathrm{r}}(x-\mu) \mathrm{d} \mu
$$

Equation 10 represents the convolution between $b(\mu)$ and $\varphi_{\mathrm{r}}(x)$, which is a particular form of Eq. 1. Thus, the same procedure proposed to solve numerically the original inverse problem can also be used here.

In fact, a similar concept has been used to characterize the IBF for the cases of FRES, NRA, RBS, and SIRM. Instead of obtaining $b(\mu)$ by solving the inverse problem stated in Eq. 10, a fixed form is assumed for $b(\mu)$, and its parameters are determined by trial and error. ${ }^{30,31} \mathrm{~A}$ Gaussian type function with a constant standard deviation, a measure of the spread of the IBF, is routinely informed as IBF for these techniques. ${ }^{1-14,23,24}$

The case of CRM deserves special attention and will be discussed in more detail. This experimental technique can be applied to study interphases by scanning the sample in the two modes described before. In the $A$ mode a bell-shaped type curve with constant width, routinely referred as the lateral resolution of CRM, has been reported as IBF. ${ }^{32-34}$ The curve is symmetrical and its tails tend to zero rather slowly. ${ }^{20-22}$ It depends on instrumental conditions such as laser wavelength, magnification of the microscope objective used, and pinhole opening, and on the quality of the optical system used. ${ }^{20-22,32-34}$ Methods proposed to measure the broadening function in this mode are based on laterally scanning the laser spot over a very sharp edge and measuring the Raman signal as a function of the position..$^{32,34,35}$ The $B$ mode is sometimes preferred to the $A$ mode because the sample does not need microtoming in order to be measured. However, broadening effects caused by the laser refraction at the sample/air interface become very important in this mode of focusing. On the one hand, refraction produces an increase in the range of depths where chemical composition is averaged as one measures deeper into the sample. On the other hand, refraction at the air/sample interface causes the actual region where local chemical composition is measured to be placed deeper than the micrometric positioning screw indication. ${ }^{18,25,26,36}$ As a result, the broadening function is a smooth but non-symmetrical curve, with spread dependent on the point where chemical composition is measured (broader as one focuses deeper into the sample). This causes a severe distortion of the chemical composition profiles, making the correction proposed essential, and besides makes the previously explained methods to obtain the IBF inapplicable. Everall ${ }^{25,26}$ and Batchelder et al. ${ }^{36}$ have proposed models that predict quite precisely the broadening function and the effect of the focusing depth displacement as a function of the depth and material properties (index of refraction), but they will not be described here. However, the determination and use of the IBF in the $B$ mode presents an interesting challenge to test the general methodology proposed here and will be explored in a future work.

\section{EXPERIMENTAL}

In this study two types of polymers were used: polystyrene (PS) and poly(phenylene oxide) (PPO). The PS sample $\left(\mathrm{Mn}=3900 \mathrm{~g} / \mathrm{mol} ; \mathrm{Mw}=4350 \mathrm{~g} / \mathrm{mol} ; T_{\mathrm{g}}=351\right.$ K) was purchased from Polymer Source (Dorval, Cana- da); the maker labeled this sample as P1779-St. The PPO sample used $(\mathrm{Mn}=15500 \mathrm{~g} / \mathrm{mol} ; \mathrm{Mw}=31000 \mathrm{~g} / \mathrm{mol}$; $T_{\mathrm{g}}=485 \mathrm{~K}$ ) was provided by General Electric. Molecular weight data were provided by the makers; the glass transition temperatures were determined by DSC using a Shimazdu DSC-50 instrument. All samples were exhaustively dried under vacuum before use to remove any traces of solvent or moisture present.

Homogeneous blends were prepared by weighing the polymers in the desired proportions, dissolving the solids in benzene at room temperature (about $10 \%(\mathrm{w} / \mathrm{v})$ solutions), and freeze-drying the necessary amounts of solutions. Blends were optically clear; DSC thermograms reveal a single $T_{\mathrm{g}}$ between the $T_{\mathrm{g}}$ values corresponding to the homopolymers.

Bilayer samples were prepared as described elsewhere. ${ }^{37}$ A thick layer (about 500 micrometers thick) was first prepared by vacuum molding a blend made out of 90/10 (w/w) PPO/PS at temperatures at least $40 \mathrm{~K}$ above the blend $T_{\mathrm{g}}$. The small quantity of PS contributes to lower the PPO $T_{\mathrm{g}}$ and overcomes the difficulties of molding pure PPO. The DSC thermogram of this blend showed a glass transition temperature at $463 \mathrm{~K}$. A thinner layer (typically between 10 and 80 micrometers thick) made out of 20/80 (w/w) PPO/PS was vacuum molded on the top of the thick layer. The DSC thermogram of this blend showed a glass transition temperature at 365 $\mathrm{K}$. Temperatures and times of molding were conveniently chosen below the thick layer $T_{\mathrm{g}}$ to minimize diffusion at this stage. These samples are labeled as type ST. For some selected samples, labeled as type DF, diffusion between layers was promoted by elevating the temperature in a controlled vacuum oven for specified periods of time.

Confocal Raman microspectroscopy (CRM) has been used to measure the local chemical composition across the bilayer interphases. Local Raman spectra were measured at room temperature on a Raman Microspectrometer DILOR LabRam Confocal, using a $16 \mathrm{~mW}$ He-Ne laser beam with a wavelength of $632.8 \mathrm{~nm}$. The pinhole opening was $700 \mu \mathrm{m}$ (the maximum aperture is 1000 $\mu \mathrm{m})$. In the excitation and collection path, an Olympus $\times 50(\mathrm{NA}=0.7)$ "dry" metallurgical objective was used. A slit opening of $500 \mu \mathrm{m}$ and a holographic grating of 1800 lines $/ \mathrm{mm}$ were used, which allows acquiring data in a Raman shift range between 500 and $1500 \mathrm{~cm}^{-1}$ with a spectral resolution of $4 \mathrm{~cm}^{-1}$. Usually, the acquisition time for each spectrum was $300 \mathrm{~s}$ and 10 spectra were accumulated for each data point.

The sample interphases were scanned operating the CRM in the $A$ mode. Previously, samples were microtomed (at room temperature) to allow us to focus the laser beam normally into the direction where chemical composition gradients are encountered. Microtoming also contributes to smoothing the sample surface, improving the Raman signal. Composition profiles at the interphase were obtained by scanning the sample interphase in steps varying between 5 and $10 \mu \mathrm{m}$ along the mass transport direction. Local chemical compositions were calculated from the acquired local Raman spectra using a linear decomposition method. ${ }^{38}$

The IBF for CRM in the $A$ mode was estimated by scanning laterally $(X$-scan) the edge of a flat and polished silicon plate in air. The intensity of the $520 \mathrm{~cm}^{-1}$ silicon 


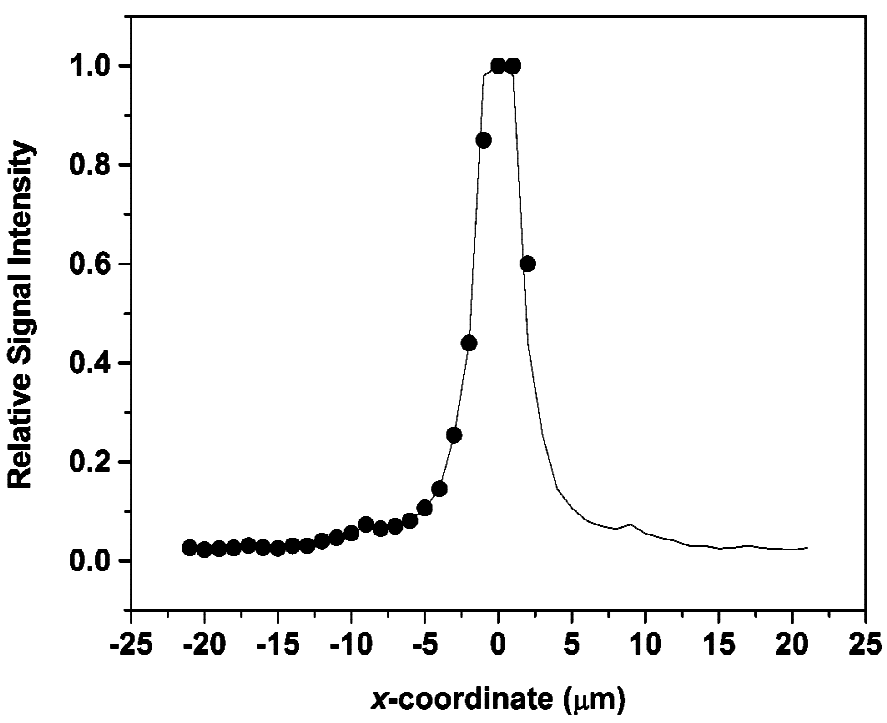

FIG. 1. Intensity of the $520 \mathrm{~cm}^{-1}$ silicon Raman band as a function of the distance from the silicon edge. Positive values in the $x$-axis correspond to measurements into the silicon plate.

line as a function of the position with respect to the edge is shown in Fig. 1 with solid circles. This curve has been determined with the same instrumental conditions (pinhole and slit opening, type of grating and objective) used for the measurements of composition profiles detailed before. The IBF used for the calculations is shown as a continuous line. The part of the IBF corresponding to distances between 0 and $20 \mu \mathrm{m}$ has been taken as the specular image of the IBF experimentally measured between -20 and $0 \mu \mathrm{m} .{ }^{21,22}$ As the limits of the silicon plate are somewhat arbitrary, these measurements can only be considered as an estimation of the lateral resolution. The nominal lateral resolution informed for the instrument in the acquisition conditions described above is on the order of 7-8 $\mu \mathrm{m} .{ }^{39}$ More precise methods to measure lateral resolution can be found in Refs. 32, 34, and 35.

\section{RESULTS AND DISCUSSION}

Numerical Verification. The technique was first tested using simulated experiments. Two cases were considered: in the first case, the real composition profile was taken as that arising from a symmetric and smooth interphase. In the second case, the composition profile at a non-symmetric interphase, which presents an abrupt change of slope, was considered. In Fig. 2 both "real" profiles, $\varphi_{\mathrm{r}}(x)$, are shown in solid lines. Notice that arbitrary units (A.U.) have been used in both coordinate and composition scales. Simulated experimental profiles, $\varphi_{\mathrm{m}}(x)$, were generated using as instrumental broadening function in Eq. 1, the function given in Eq. 8 with:

$$
b(\mu-x)=\frac{1}{\sigma \sqrt{2 \pi}} e^{\left[-(x-\mu)^{2} / 2 \sigma^{2}\right]}
$$

This is the normal probability density function. $\mu$ is the mean of the density function, and $\sigma$, the standard deviation, is a measure of the spread of the broadening function. This function is also plotted in Fig. 2 for $\mu=60$ and $\sigma=7$ as a dotted line. In this example the value of $\sigma$ has been chosen to generate an interphase with a degree

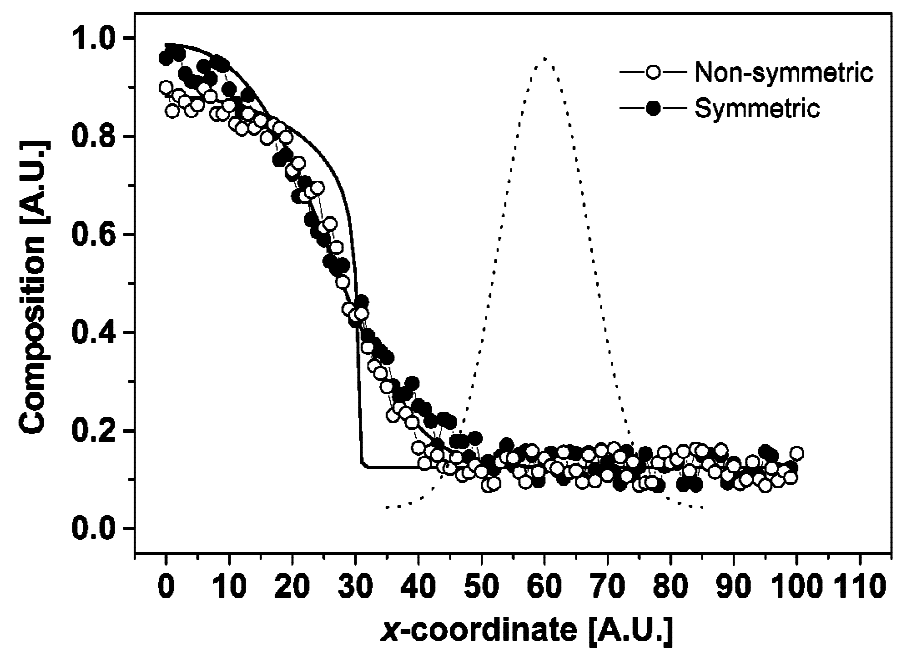

FIG. 2. Simulated chemical composition profiles for both symmetric and non-symmetric interphases (filled and open symbols). The true profiles are shown in full lines. The bell-shaped curve represents the IBF used for the simulations for $\mu=60$ and $\sigma=7$.

of blurring similar to the one found in real experiments. The value of $\sigma=7$ used in the calculations gives a broadening function with a full width at half-maximum (FWHM) of 14 A.U. $(\sim 2 \sigma)$. The simulated experimental profiles computed with Eq. 1 were artificially distorted with measurement noise to imitate a real situation. They are shown in Fig. 2 as open and filled circles.

It is interesting to note that both "experiments" are quite similar, which reveals the lack of capability of the untreated measurements to distinguish between symmetrical and non-symmetrical interphases.

Before proceeding with the numerical treatment of the "experimental" data, matrix $\boldsymbol{\Gamma}$ must be selected. This matrix, which ponders the relative amount of regularization along the $x$ coordinate, is chosen to be a diagonal matrix with elements that make regularization strong where the signal is flat, and weak where the signal changes rapidly. Thus, the diagonal elements of $\boldsymbol{\Gamma}$, i.e., $\gamma_{1}, \gamma_{2}, \ldots, \gamma_{n_{2}}$, are sampled from the following function:

$$
\gamma_{i}=1-\exp \left[\frac{-(i-m)^{2}}{2 \sigma_{\gamma}^{2}}\right] \quad i=1, \ldots, n_{2}
$$

where $m$ is the row of $\boldsymbol{\Gamma}$ that corresponds to the maximum change of the measured signal. $\sigma_{\gamma}$ is a measure of how fast regularization is increased to both sides of the point of maximum change. Note that for $i=m$ (the point of maximum change), regularization is null, while for values of $i$ far from $m$ the elements of $\boldsymbol{\Gamma}$ tend to 1, meaning maximum regularization in the regions where the signal is known to be smooth. In these examples $\sigma_{\gamma}=20$ and the point of maximum change, computed numerically from the distorted signal, corresponds to the row of $\boldsymbol{\Gamma}$ associated with $x=30$ on the $x$ axis. The results showed to be not very sensitive to the value of $\sigma_{\gamma}$.

In Fig. 3 the corrected profile computed using Eq. 6 is shown together with the real one for the example with a symmetrical interphase. In Fig. 4 the corrected profile for the non-symmetric interphase is also shown, together with the real one. In both cases the corrected profiles are very much like the real ones. In these examples the val- 


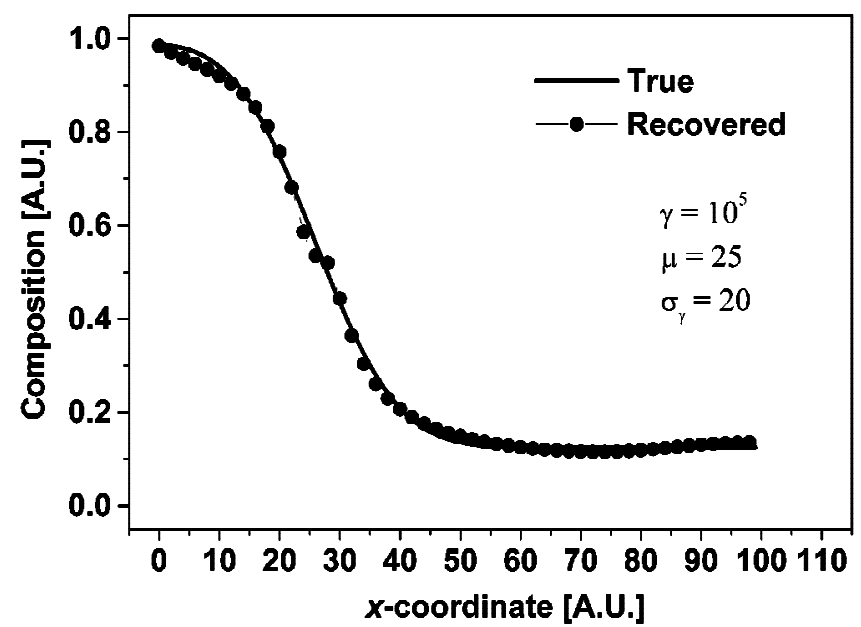

FIG. 3. Recovered and expected chemical composition profiles for a smooth and symmetric interphase. The value of the $\gamma$ parameter is determined by trial and error.

ues of $\gamma$ were selected by trial and error and represent the optimum choice, only possible if the solution is known. In a real case the GCV technique described above must be used to estimate $\gamma$ from the data. The result for the non-symmetric interphase is shown in Fig. 5. It can be noticed that the automatic determination of $\gamma$ produces some degradation of the computed profile with respect to the optimum solution. However, the main features of the interphase are still recovered despite the spurious oscillations that appear to the right of the contact point. These oscillations are due to an underestimation of $\gamma$, which is characteristic in all cross-validation methods.

Application to Genuine Experimental Data. The technique is now applied to recover the "true" composition profile from real CRM measurements. As smooth composition profiles are much less affected by the instrumental broadening, the technique was tested only on samples that present marked changes of composition across the interphase.

Figures 6A and 6B show (with solid lines) composition profiles at the interphase (from CRM measurements) for the samples labeled as ST1 and ST2. These samples are prepared by putting in contact two polymer layers with different chemical compositions through a planar interface, as detailed in the Experimental section. The thin layer was molded on top of the thick layer at $393 \mathrm{~K}(28$ $\mathrm{K}$ above the thin layer $T_{\mathrm{g}}$, and $70 \mathrm{~K}$ below the thick layer $T_{\mathrm{g}}$ ), for about $15 \mathrm{~min}$. Under these conditions the diffusion coefficients for the mobile thin layer (low $T_{\mathrm{g}}$ blend) in the glassy thick layer (high $T_{\mathrm{g}}$ blend) lower than $10^{-20}$ $\mathrm{cm}^{2} / \mathrm{s}$ are expected. ${ }^{5,6}$ Any calculation will show that the diffusion caused by this sample preparation should not be observed in the abscissa scale of Figs. 6A-6B. Very sharp composition profiles at the interphase, like a step profile, are expected for these samples, with only minor rugosities as mentioned before by other authors. ${ }^{39}$ Instead of that, experimental profiles appear blurred, due to the poor spatial resolution.

Filled circles represent the composition profiles recovered once the proposed technique was applied. For the calculations, the instrumental broadening function shown in Fig. 1 was assumed. The $\boldsymbol{\Gamma}$ matrix is selected as was

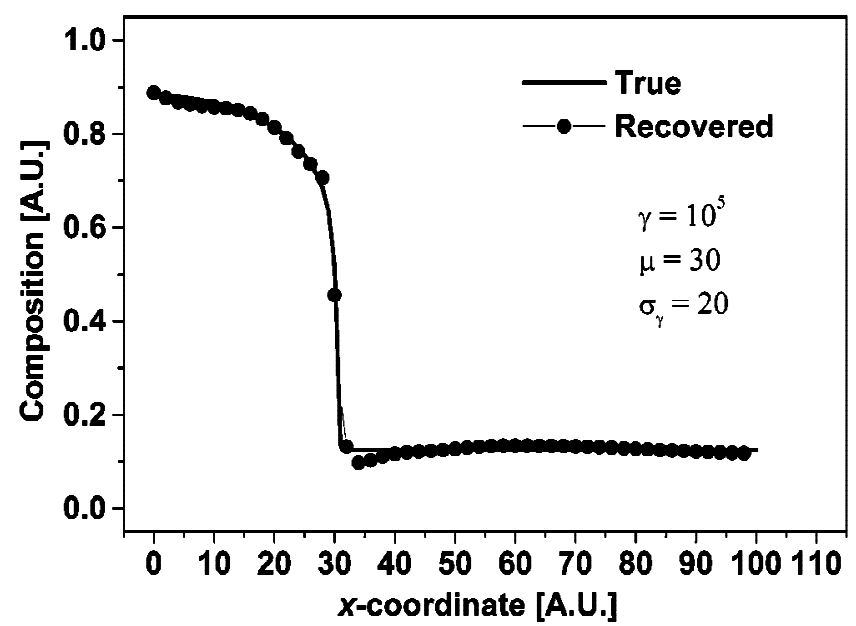

FIG. 4. Recovered and expected chemical composition profiles for an abrupt and non-symmetric interphase. The value of the $\gamma$ parameter is determined by trial and error.

detailed in the Numerical Verification subsection. The values of the regularization parameter were automatically selected using the GCV method $\left(\gamma=10^{-2}\right)$. The recovered profiles very closely resemble step profiles, as expected. As a guide, these step profiles are shown in the figures (as dotted lines) and coincide quite well with the recovered profiles, taking into account the limitation in the precision of the estimated IBF.

Figures 7A and 7B show the experimentally measured composition profiles using CRM for the samples labeled as DF1 and DF2. These samples have been subjected to a thermal treatment that promotes mass transport between the layers. The layers have very different physical properties (see $T_{\mathrm{g}}$ values of each layer in the Experimental section); it is well established that diffusion will generate markedly non-symmetric composition profiles at the polymer-polymer interphase for this case..$^{5,6,9,18} \mathrm{Compo-}$ sition profiles that present higher slopes located near to the high $T_{\mathrm{g}}$ layer, and a gradually decreasing slope towards the region of the low $T_{\mathrm{g}}$ layer have been observed for this type of polymer pair., ${ }^{9,16}$ The shape of these profiles can be precisely calculated using well-established

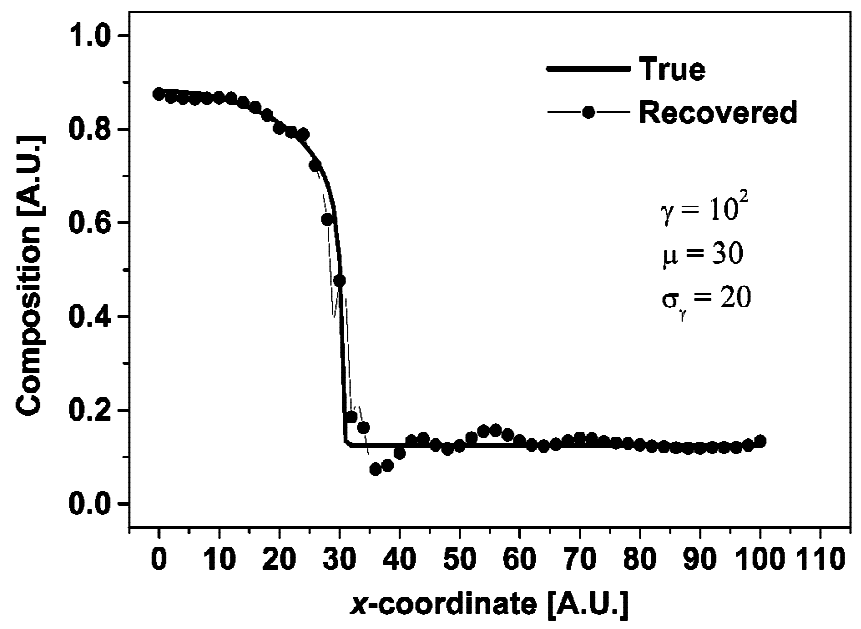

FIG. 5. Recovered and expected chemical composition profiles for an abrupt and non-symmetric interphase. The value of the $\gamma$ parameter is determined using the GVC method. 
A
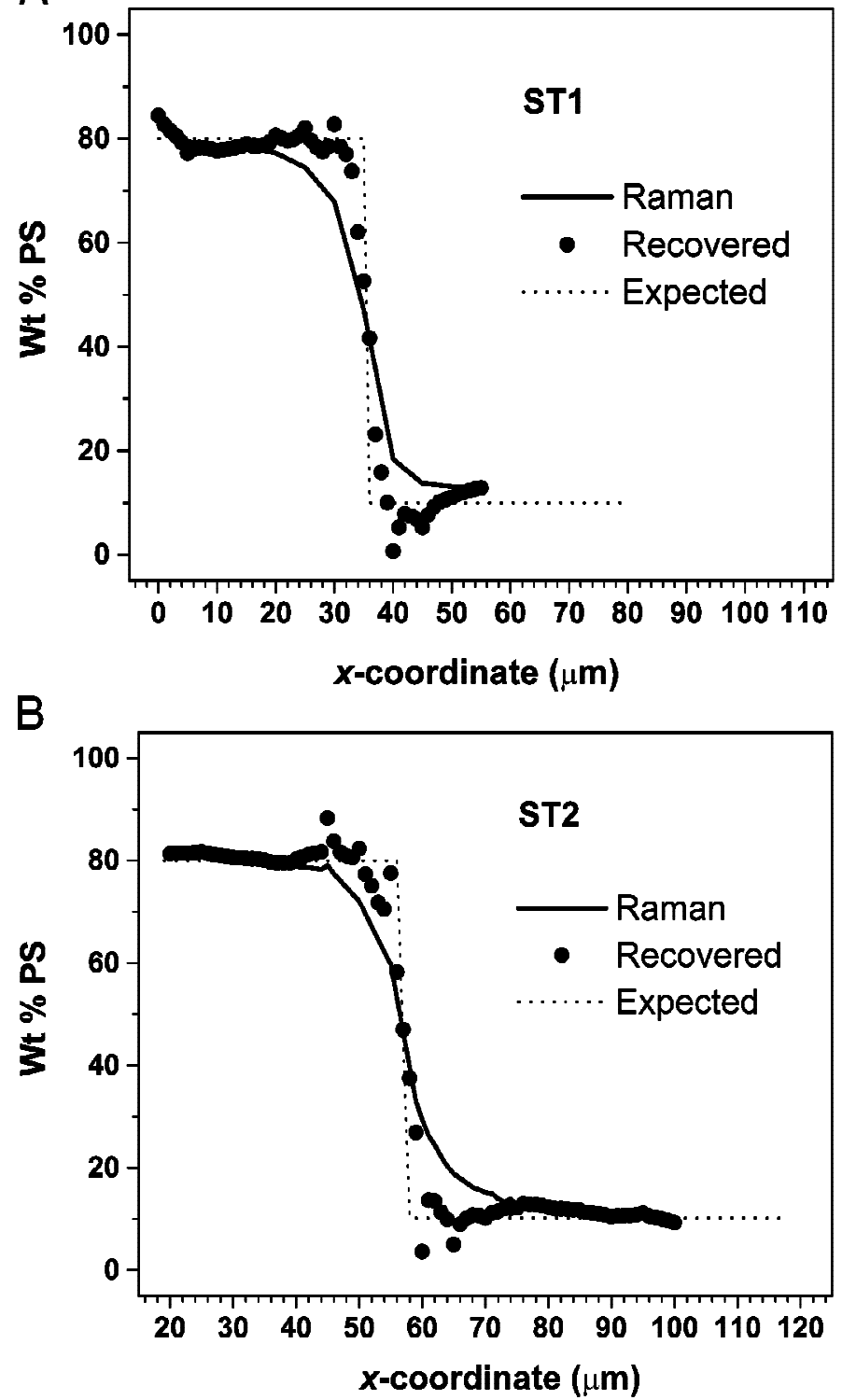

FIG. 6. Experimentally measured chemical composition profiles at the interphase for the bilayer polymer samples labeled as (A) ST1 and (B) ST2 (full lines). The recovered profile is represented with solid circles. The value of the $\gamma$ parameter is determined using the GVC method. Expected profiles are shown as a guide with dotted lines.

models for polymer interdiffusion. ${ }^{15,16}$ Interphase composition profiles calculated using the above-mentioned models are also shown in the figures (dotted lines) as a guide. It can be observed that the models predict profiles with a planar and very sharp interphase, without tails. Details of the calculations can be found elsewhere and are out of the scope of this work. ${ }^{41}$

The experimentally measured interphase composition profiles are shown in solid lines in Figs. 7A and 7B. Even though they present the above-mentioned characteristics, they also show "tails" ahead of the diffusion front due to the poor spatial resolution. The presence of tails is a distinctive feature of the occurrence of certain diffusion mechanisms (i.e., Case II diffusion regime ${ }^{2-4,7-11}$ ), and it is very important to discern whether tails are produced by genuine mass transport or are a purely instrumental
A

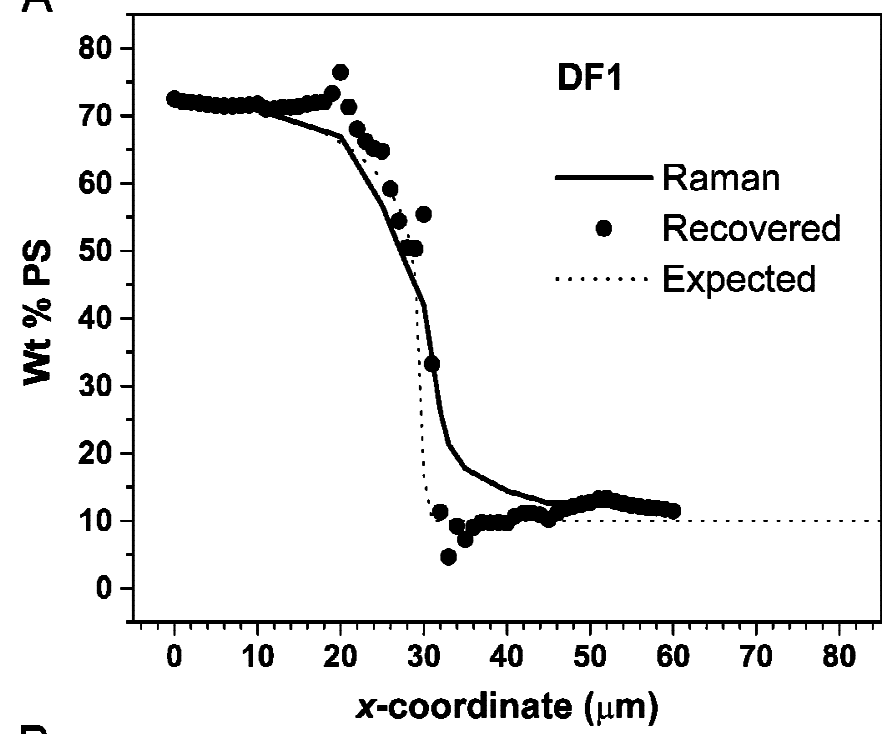

B

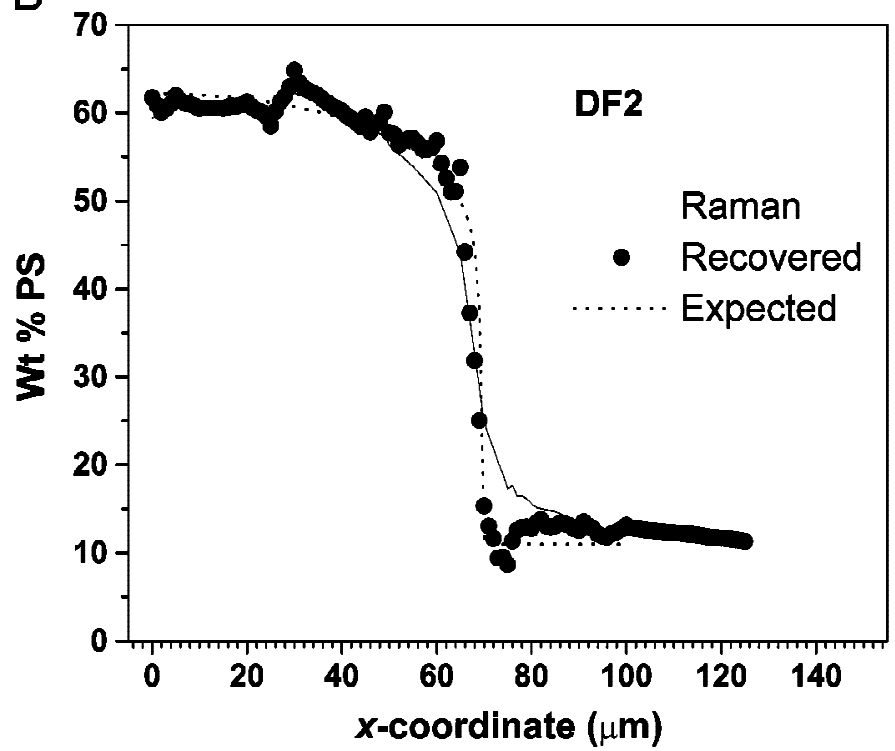

FIG. 7. Experimentally measured chemical composition profiles at the interphase for the bilayer polymer samples labeled as (A) DF1 and (B) DF2 (full lines). The recovered profile is represented with solid circles. The value of the $\gamma$ parameter is determined using the GVC method. Expected profiles calculated using diffusion models are shown as a guide with dotted lines.

artifact. The recovered composition profiles calculated using the proposed technique are shown in these figures in solid circles $\left(\gamma=5 \times 10^{-2}\right)$. The tails observed in the experimental data behind the diffusion front are completely eliminated, suggesting that they are spurious and produced by the instrumental broadening. The recovered profiles present sharp diffusion fronts, as expected for this polymer pair, and they coincide quite precisely with the calculated ones.

\section{CONCLUSION}

An inversion technique based on Phillips-Thikonov regularization was successfully applied to correct experimentally measured chemical composition profiles at polymer interphases. Corrections are needed because of 
the rounding effects caused by the limited resolution of the commonly used experimental techniques.

The importance of the correction was more clearly evidenced in the case of unsymmetrical interphases or in cases in which sharp diffusion fronts were present. The proposed correction may help to differentiate between profiles with tails produced by genuine mass transport and profiles in which tails are a purely instrumental artifact.

The correction proposed does not require any previous information related to the sought profile other than the experimental data. The model of the measurement process to be inverted includes what is called here the IBF, which represents the influence of the instrumental broadening on the measurement. This function must either be determined experimentally or estimated. It is in most cases invariant (i.e., not dependent on the position in the sample), and this is the case considered in this work. A remarkable exception is CRM in the depth profiling mode where the broadening varies with the position in the sample (the deeper the broader).

To test the methodology and to show how it works, the method was applied to experimental runs obtained using CRM in the $A$ mode, where IBF can be considered invariant, using acquisition conditions that increase the broadening. The method was able to recover profiles free of instrumental effects, founding good agreement between the recovered profiles and the expected ones, keeping in mind the limitation in the estimation of the IBF. Even though in this specific case (CRM in surface-profiling mode) it is possible to prevent the blurring by choosing better instrumental conditions for data acquisition, in other cases (depth-profiling mode using CRM instruments equipped with metallurgical "dry" objectives) this is difficult to achieve due to the refraction at the sample/air interface. ${ }^{17,18,25,26,36} \mathrm{We}$ expect that this methodology will be especially useful in the latter case. What is challenging in the depth-profiling mode is that blurring increases with the position in the sample. However, the methodology presented here does not hold this condition and will be treated in future work.

\section{ACKNOWLEDGMENTS}

Pablo Tomba would like to thank Professor José M. Pastor (Departamento de Física del la Materia Condensada, Universidad de Valladolid, Spain) for the use of the Raman Microspectrometer and Dr. David G. Lopez for his valuable assistance during the measurements.

1. P. J. Mills, P. F. Green, C. J. Palmstroom, J. W. Mayer, and E. J. Kramer, J. Polym. Sci.: Polym. Phys. Ed. 24, 1 (1986).

2. C.-Y. Hui, K.-C. Wu, R. C. Lasky, and E. J. Kramer, J. Appl. Phys 61, 5129 (1987)
3. C.-Y. Hui, K.-C. Wu, R. C. Lasky, and E. J. Kramer, J. Appl. Phys 61, 5137 (1987).

4. R. C. Lasky, E. J. Kramer, and C.-Y. Hui, Polymer 29, 673 (1988).

5. R. J. Composto, E. J. Kramer, and D. M. White, Macromolecules 21, 2580 (1988)

6. R. J. Composto, E. J. Kramer, and D. M. White, Polymer 31, 2320 (1990).

7. T. P. Gall, R. C. Lasky, and E. J. Kramer, Polymer 31, 1491 (1990).

8. T. P. Gall and E. J. Kramer, Polymer 32, 265 (1991).

9. R. J. Composto and E. J. Kramer, J. Mater. Sci. 26, 2815 (1991).

10. P. F. Nealey, R. E. Cohen, and A. S. Argon, Polymer 36, 3687 (1995).

11. Q.-Y. Zhou, A. S. Argon, and R. E. Cohen, Polymer 42, 613 (2001).

12. U. Steiner, U. K. Chaturvedi, O. Zak, G. Krausch, G. Schatz, and J. Klein, Makromol. Chem., Makromol. Symp. 45, 283 (1991).

13. A. Losch, D. Woermann, and J. Klein, Macromolecules 27, 5713 (1994).

14. T. E. Shearmur, A. S. Clough, D. W. Drew, M. G. D. van der Grinten, and R. A. L. Jones, Macromolecules 29, 7269 (1996).

15. J. P. Tomba and J. M. Carella, J. Polym. Sci.: Polym. Phys. Ed. 37, 3097 (1999).

16. J. P. Tomba, J. M. Carella, D. García López, and J. M. Pastor, Macromolecules 21, 235 (2001).

17. S. Hajatdoost and J. Yarwood, Appl. Spectrosc. 50, 558 (1996).

18. S. Hajatdoost, M. Olsthoorn, and J. Yarwood, Appl. Spectrosc. 51, 1784 (1997).

19. J. Sacristán, C. Mijangos, H. Reinecke, S. Spells, and J. Yarwood, Macromolecules 33, 6134 (2000).

20. R. J. Meier and B. J. Kip, Microbeam Analysis 3, 61 (1994).

21. R. Tabaksblat, R. J. Meier, and B. J. Kip, Appl. Spectrosc. 46, 60 (1992).

22. A. Garton, D. N. Batchelder, and C. Cheng, Appl. Spectrosc. 47, 922 (1993)

23. E. A Jordan, R. C. Ball, A. M. Donald, L. J. Fetters, R. A. L. Jones, and J. Klein, Macromolecules 21, 235 (1988).

24. J. v. Seggern, S. Klotz, and H. J. Cantow, Macromolecules 22, 3328 (1989).

25. N. J. Everall, Appl. Spectrosc. 54, 773 (2000).

26. N. J. Everall, Appl. Spectrosc. 54, 1515 (2000).

27. D. L. Phillips, J. AMC 9, 84 (1962).

28. G. E. Eliçabe and G. Rubio, L. Adv. Chem. Ser. 227, 83 (1990).

29. G. H. Golub, M. Heath, and G. Wahba, Technometrics 21, 215 (1979).

30. P. J. Mills, P. F. Green, C. J. Palmstroom, J. W. Mayer, and E. J. Kramer, Appl. Phys. Lett. 45, 957 (1984).

31. U. K. Chaturvedi, U. Steiner, O. Zak, G. Krausch, G. Schatz, and J. Klein, Appl. Phys. Lett. 56, 1228 (1990).

32. G. P. Puppels, W. Colier, J. H. F. Olminkhof, C. Otto, F. F. M. de Mul, and J. Greeve, J. Raman Spectrosc. 22, 217, (1991).

33. T. Wilson, J. Microscopy 154, 143 (1989).

34. G. J. Brakenhoff, P. Blom, and P. Barends, J. Microscopy 117, 219 (1979).

35. D. J. Gardiner, M. Bowden, and P. R. Graves, Philos. Trans. R. Soc. London, Ser. A 320, 295 (1986).

36. K. J. Baldwin and D. N. Batchelder, Appl. Spectrosc. 55, 60 (2001).

37. J. P. Tomba, J. M. Carella, and E. Pardo, J. Polym. Sci., Part B: Polym. Phys. 35, 2435 (1997).

38. J. P. Tomba, E. De la Puente, and J. M. Pastor, J. Polym. Sci., Part B: Polym. Phys. 38, 1013 (2000).

39. LabRam Users Manual (Dilor, 1997).

40. E. Jabbari and N. A. Peppas, Macromolecules 26, 6229 (1993).

41. J. P. Tomba, J. M. Carella, J. M. Pastor, and J. C. Merino, Polymer 43, 6751 (2002). 